\title{
S100 and p65 expression are increased in the masseter muscle after botulinum toxin-A injection
}

\author{
Young-Wook Park', Seong-Gon Kim"id and You-Young Jo²
}

\begin{abstract}
Background: The purpose of this study was to compare the expression levels of p65 and S100 in the rat masseter muscle after the injection of different concentrations of botulinum toxin-A (BTX-A).

Methods: We injected either 5 or $10 \mathrm{U}$ of BTX-A into both masseter muscle of rats. As a control group, the same volume of saline was injected. After 14 days, the animals were sacrificed. Subsequently, a biopsy and immunohistochemical staining of the samples were performed using a p65 or \$100 antibody.

Results: The cross-sectional area of each myofibril was significantly reduced by BTX-A injection $(P<0.001)$. The expression of p65 and S100 increased significantly with increasing concentrations of BTX-A $(P<0.001)$.

Conclusions: The injection of BTX-A into the masseter muscle induced muscle atrophy. Subsequently, p65 and S100 expression in myoblasts were increased for the protection of muscle cells.
\end{abstract}

Keywords: Botulinum toxin-A, p65, S100, Apoptosis, Masseter muscle

\section{Background}

Botulinum toxin is a neurotoxin produced by the anaerobic bacterium Clostridium botulinum [1, 2]. This toxin selectively hydrolyzes the receptor that is required for the binding of the synapse vesicle and the membrane on the pre-synapse of the neuromuscular junction; as a result, the toxin blocks the release of acetylcholine [3-6], causing muscle weakness, paralysis, and atrophy [7]. Botulinum toxin-A (BTX-A) is a type of botulinum toxin that was approved by the US Food and Drug Administration (FDA) in 1989. Since that time, BTX-A has been used for cosmetology, focal dystonia, facial spasm, hyperhidrosis, and the treatment of muscle hypertrophic disorder $[8,9]$.

In addition, in the oral and maxillofacial area, BTX-A is injected into masticatory muscles such as the masseter muscle or the temporal muscle for esthetic and therapeutic purposes [10-12]. Previous studies primarily investigated the effect of BTX-A on muscles and nerves

\footnotetext{
* Correspondence: kimsg@gwnu.ac.kr

${ }^{1}$ Department of Oral and Maxillofacial Surgery, College of Dentistry,

Gangneung-Wonju National University, 7 Jukhyun-gil, Gangneung 210-702,

South Korea

Full list of author information is available at the end of the article
}

[13]. However, muscle paralysis or weakness impacts on the bone, resulting in a reduction of the affected bone, even if BTX-A is injected into the muscle $[14,15]$. Therefore, more studies are needed to explain the mechanism by which BTX-A affects the masseter muscle.

BTX-A injection into the masseter muscle induces muscle atrophy [16]. Several marker proteins have the potential to increase their expression during the skeletal muscle atrophy process [17]. S100 is a well-known calcium-binding protein $[18,19]$. The terminal deoxynucleotidyl transferase dUTP nick end labeling (TUNEL) assay has been used to assess apoptosis in many publications [20]. After burn injury, muscle atrophy is accompanied by increasing S100 levels and TUNEL-positive cells in the motor nerve [21]. The NF-kB pathway is important in cellular apoptosis. The expression of caspases and key components of the NF-kB pathway (p65) is increased during skeletal muscle atrophy [22]. Therefore, the expression levels of S100 and p65 in the masseter muscle may change after BTX-A injection. However, S100 expression levels have not been studied before in the context of BTX-A injection. 
The purpose of this study was to compare the expression levels of p65 and S100 in the rat masseter muscle after the injection of different concentrations of BTX-A.

\section{Methods}

\section{Experimental animals and housing conditions}

Fifteen Sprague-Dawley rats (age 10-12 weeks, body weights 250-300 g) were purchased from Samtako (Osan, Korea). Each rat was individually housed and allowed an adaptation period for 10 days. The Institutional Animal Care and Use Committee of GangneungWonju National University approved this experiment (GWNU 2015-25).

\section{Experimental design}

The animals were divided into three groups: the control group and two experimental groups. Five rats were used in each group. In one experimental group, $5 \mathrm{U}$ of a BTX-A solution was injected into both sides of the masseter muscle. In the other experimental group, $10 \mathrm{U}$ of a BTX-A solution was injected on both sides of the masseter muscle. The control group was injected with $0.1 \mathrm{cc}$ of saline on both sides of the masseter muscle. Fourteen days after injection, all rats were sacrificed, and samples containing the masseter muscle and mandible were obtained for histological examination. After the harvested tissues were fixed in a formalin solution for 1 day, decalcification was performed with a $5 \%$ nitric acid solution, and specimens containing the masseter muscle and mandible were generated. For following evaluation, the specimens were cut as horizontal plane. The height for sectioning ramus was determined as the central cut between the zygomatic arch plane and the lower mandibular border plane.

The specimens were stained with hematoxylin and eosin $(\mathrm{H} \& \mathrm{E})$ to evaluate the cortical bone thickness of the mandibular ramus. We photographed the histological view at 14 days post-surgery and measured the ramal cortical bone thickness of the rats using size measuring software (SigmaScan-Pro ${ }^{\circ}$; SPSS Science, Chicago, IL, USA). The cross-sectional area of myofibrils was also measured using the same software. Immunohistochemical staining was performed for p65 and S100. Both antibodies were murine monoclonal antibodies purchased from Santa Cruz Biotech (Santa Cruz, CA, USA). The primary antibody dilutions were as follows: S100, 1:30; and p65, 1:50. Subsequent procedures were in accord with a previous publication [23].

To quantify the intensity of the immunohistochemical reaction, the intensity of positive staining was evaluated in five random fields of the masseter muscle at a $\times 200$ magnification using a computer-assisted image analysis program. The staining intensity in immunohistochemistry experiments was shown as the mean intensity value (0: no stain, 255: highest stain). Counterstaining procedure was omitted to ensure that the intensity value would
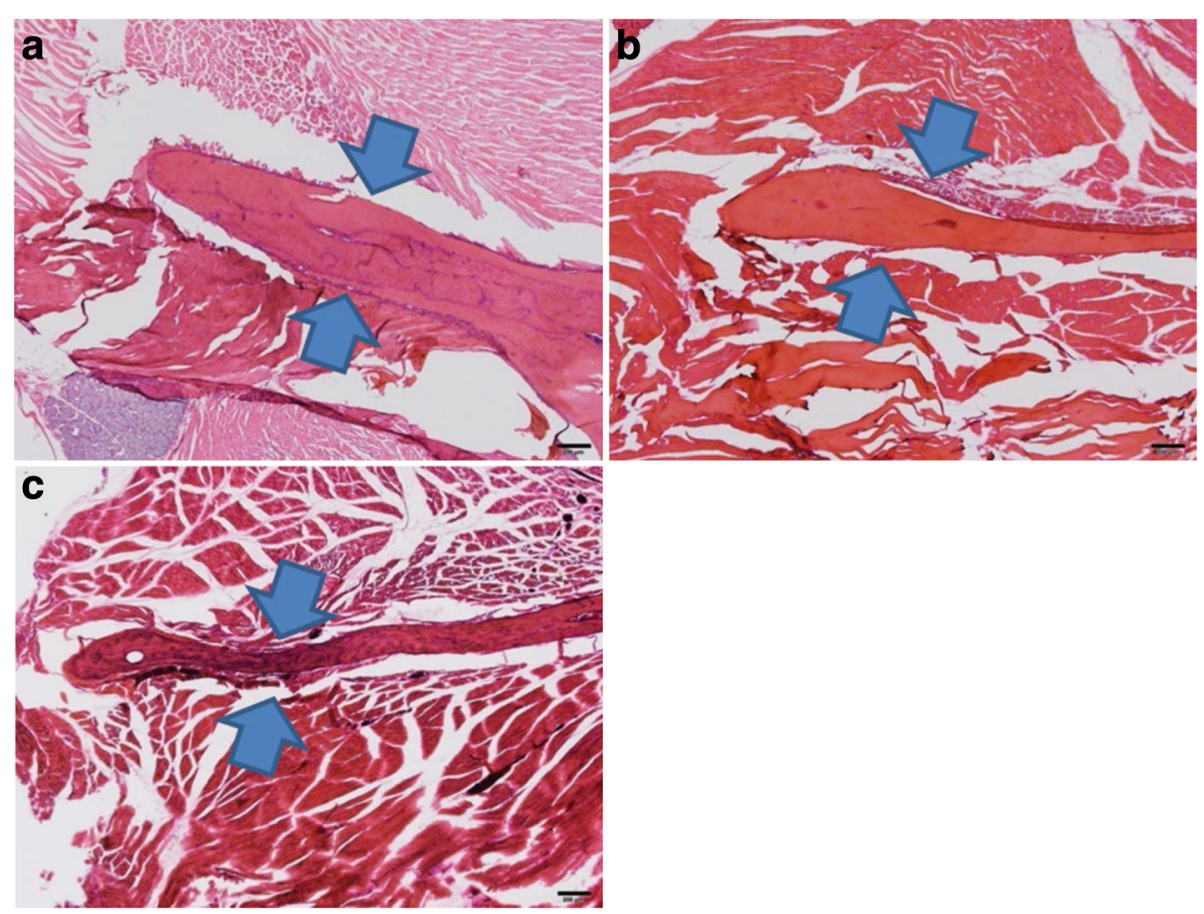

Fig. 1 Cortical bone of the rat mandibular ramus. The arrows indicate the mandibular ramus. a Saline-treated group; b 5-U BTX-A-treated group; c 10-U BTX-A-treated group (H\&E stain, original magnification $\times 20$ ) 
Table 1 The cortical bone thickness and the cross-sectional areas of masseter muscle after BTX-A injection

\begin{tabular}{llllll}
\hline Group & \multicolumn{2}{l}{ Cortical bone thickness } & & \multicolumn{2}{c}{ Cross-sectional area } \\
\cline { 2 - 3 } & Average $(\mathrm{mm})$ & $P$ value & & Average $\left(\mu \mathrm{m}^{2}\right)$ & $P$ value \\
\hline Saline-treated & $0.18 \pm 0.05$ & - & & $621.68 \pm 87.74$ & - \\
5-U BTX-A-treated & $0.15 \pm 0.05$ & NS & & $495.42 \pm 110.15$ & 0.037 \\
10-U BTX-A-treated & $0.10 \pm 0.03$ & NS & & $263.05 \pm 115.31$ & $<0.001$
\end{tabular}

The $P$ value was calculated by post hoc test and compared with the saline-treated group

$B T X-A$ botulinum toxin- $A$

be solely attributable to the positive immunohistochemical reaction.

\section{Statistical analysis}

All of the results were statistically analyzed using oneway analysis of variance (ANOVA), followed by a post hoc test (Bonferroni's method). The significance level was set as $P<0.05$.

\section{Results}

A horizontal cut of the mandibular ramus is shown in Fig. 1a-c for saline-treated, 5-U BTX-A-treated, and 10U BTX-A-treated specimens, respectively. The average thicknesses of the cortical bone were measured as follows: the saline-treated control group had a thickness of $0.18 \pm 0.05 \mathrm{~mm}$, the 5 -U BTX-A-treated group had a thickness of $0.15 \pm 0.05 \mathrm{~mm}$, and the 10-U BTX-A- treated group had a thickness of $0.10 \pm 0.03 \mathrm{~mm}$ (Table 1). The cortical bone thickness of the mandibular ramus of rats was reduced in the BTX-A-treated groups in comparison to the saline-treated control group. However, the difference observed among the groups was not statistically significant $(P=0.055)$.

A cross section of the masseter muscle is shown in Fig. 2a-c for saline-treated, 5-U BTX-A-treated, and 10-U BTX-A-treated specimens, respectively. The average crosssectional areas of myofibrils were $621.68 \pm 87.74 \mu \mathrm{m}^{2}$, $495.42 \pm 110.15 \mu^{2}$, and $263.05 \pm 115.31 \mu^{2}$ for the saline-treated control, the 5-U BTX-A-treated group, and the 10-U BTX-A-treated group, respectively (Table 1). The cross-sectional area of myofibrils was significantly reduced in the BTX-A-treated groups in comparison to the saline-treated control group $(P<$ 0.001 ). The post hoc test revealed differences between the group treated with $10 \mathrm{U}$ BTX-A and the other groups, with significantly lower values in the 10-U BTX-A group than in the saline-treated control and the 5-U BTX-A-treated group $(P<0.001$ for both groups). When we compared the 5-U BTX-A-treated group to the saline-treated control, the observed difference was statistically significant $(P=0.037)$.

The immunohistochemical findings for p65 and S100 are shown in Figs. 3 and 4. The immunohistochemical findings demonstrated that the expression of p65 and S100 were significantly higher in the 10-U BTX-A-
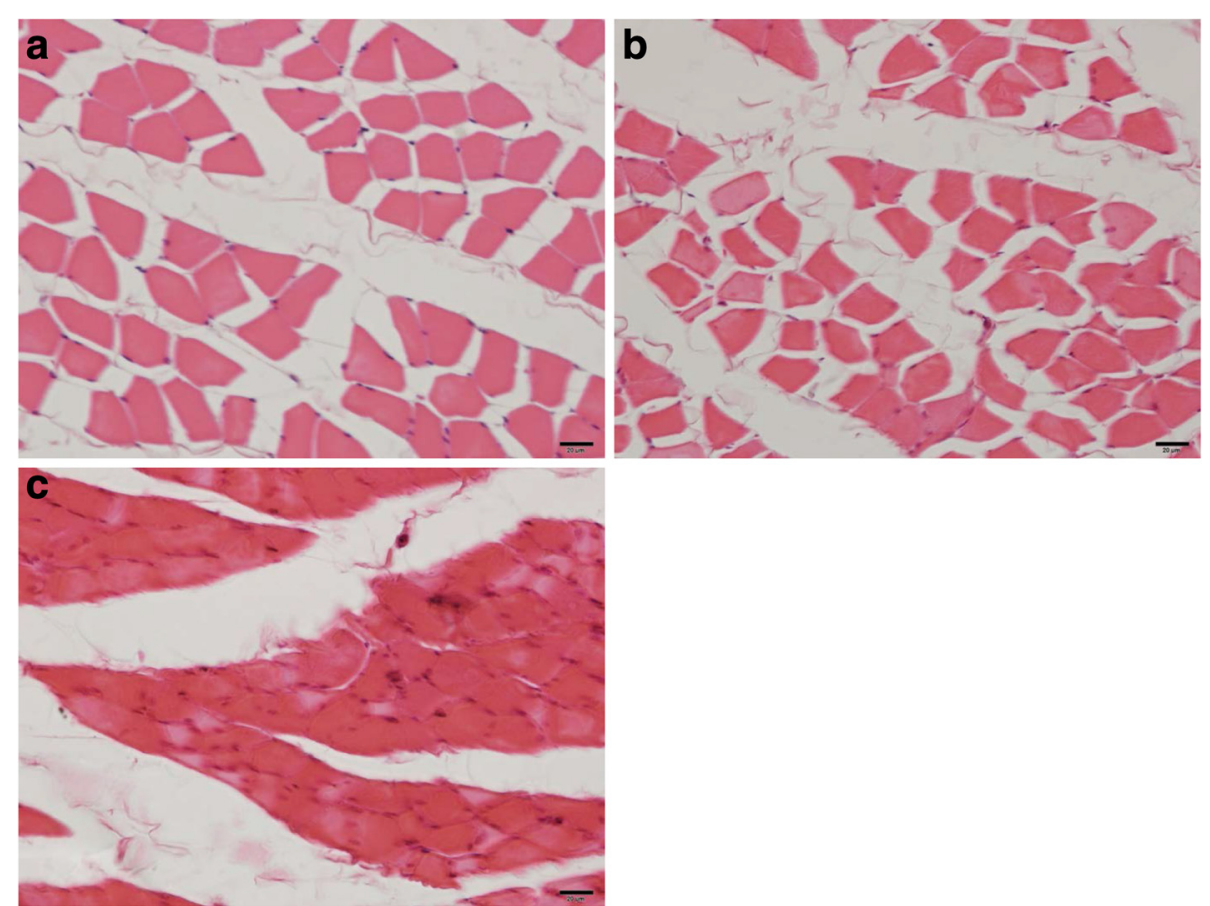

Fig. 2 Cross section of the masseter muscle. a Saline-treated group; b 5-U BTX-A-treated group; c 10-U BTX-A-treated group (H\&E stain, bar $=20 \mu \mathrm{m})$ 

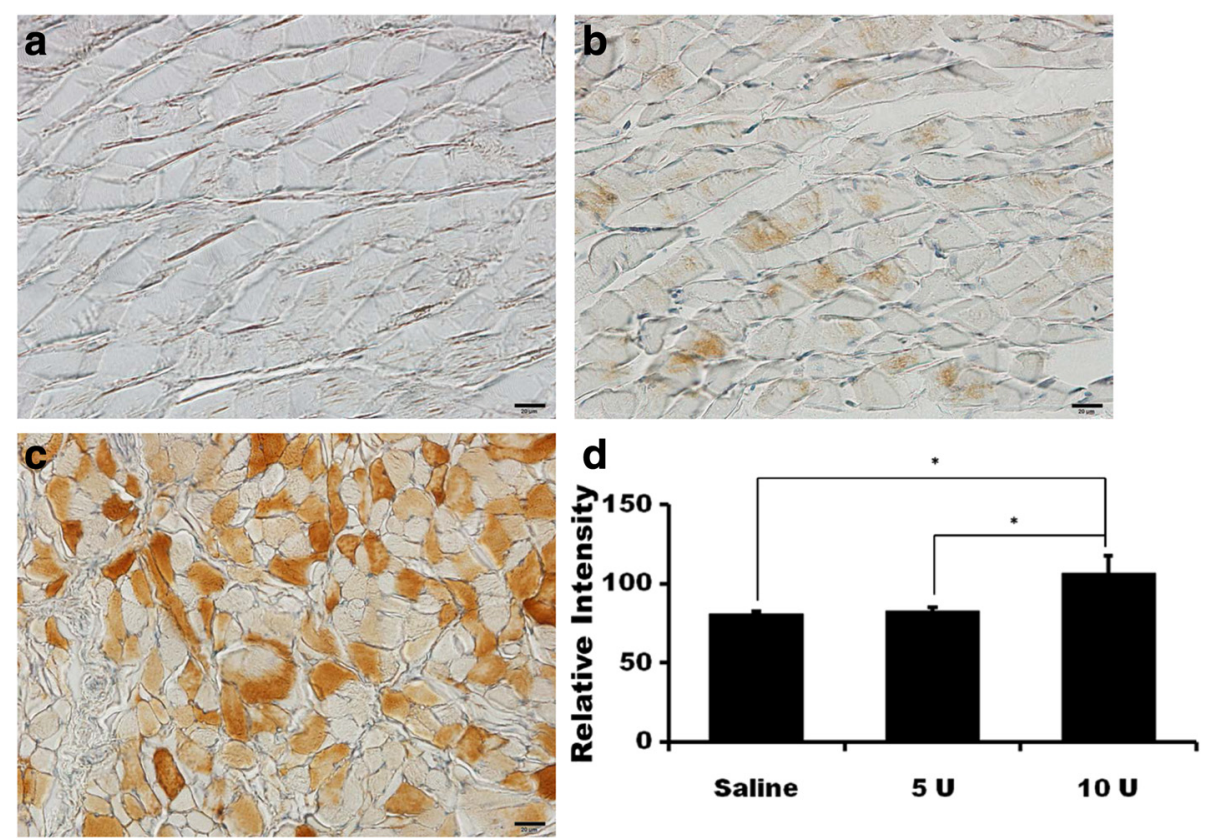

Fig. 3 Immunohistochemical staining for p65. a Saline-treated group; b 5-U BTX-A-treated group; c 10-U BTX-A-treated group (without counterstaining, $\mathrm{bar}=20 \mu \mathrm{m})$. $\mathbf{d}$ Measurement of the average intensity of staining $\left({ }^{*} P<0.05\right)$

treated groups than in the saline group (Figs. $3 \mathrm{~d}$ and $4 \mathrm{~d}$; $P<0.001$ and $P=0.002$ for $\mathrm{p} 65$ and S100, respectively). The mean intensity values for p65 were $81.02 \pm 1.53$, $82.93 \pm 2.11$, and $106.46 \pm 10.86$ for the saline, 5 -U BTXA, and 10-U BTX-A treatments, respectively. The post hoc test revealed differences between the group treated with $10 \mathrm{U}$ of BTX-A and the other groups, with significantly higher values in the 10-U BTX-A group than in the saline-treated control and the 5-U BTX-A-treated group $(P<0.001$ for both groups). The mean intensity values for $S 100$ were $78.98 \pm 4.36,85.31 \pm 3.09$, and $96.46 \pm 8.98$ for the saline, 5-U BTX, and 10-U BTX
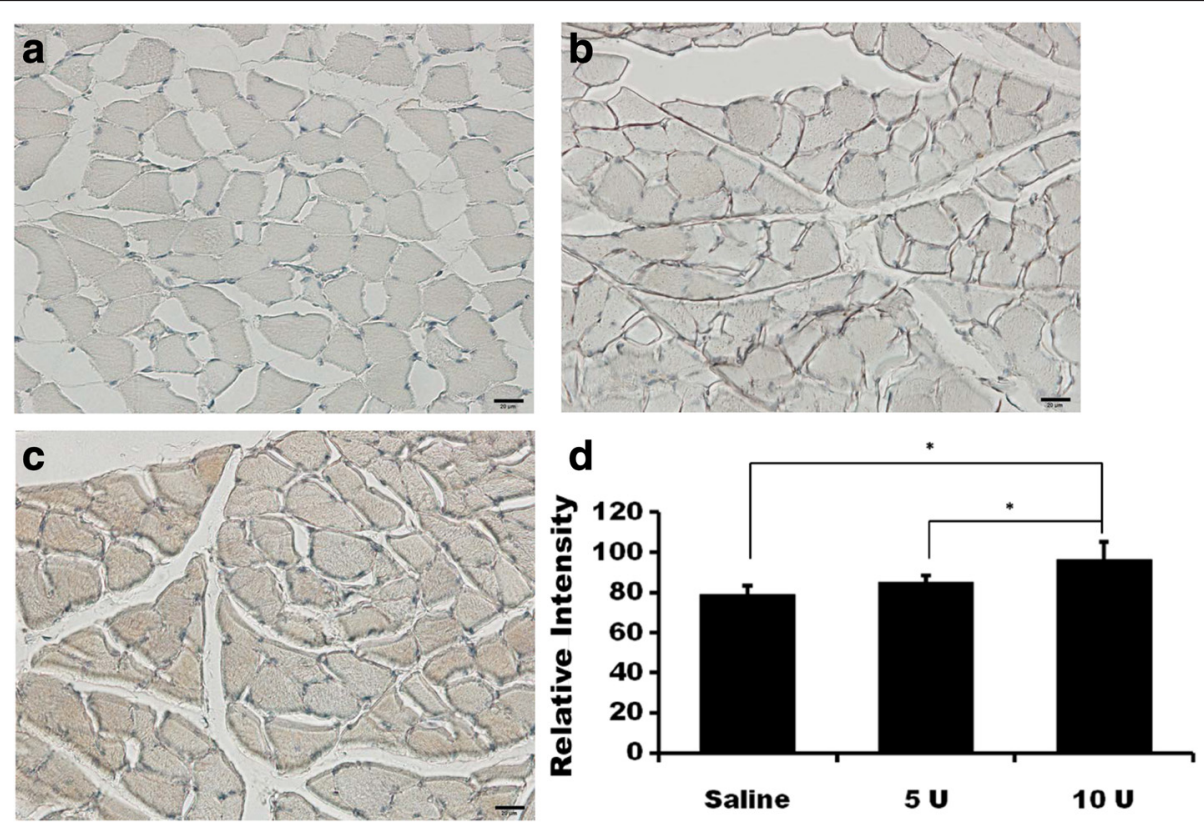

Fig. 4 Immunohistochemical staining for S100. a Saline-treated group; b 5-U BTX-A-treated group; c 10-U BTX-A-treated group (without counterstaining, bar $=20 \mu \mathrm{m}) . \mathbf{d}$ Measurement of the average intensity of staining $\left({ }^{*} P<0.05\right)$ 
treatments, respectively. The post hoc test revealed differences between the group treated with 10 U BTX-A and the other groups, with significantly higher values in the 10-U BTX-A group than in the saline-treated control and the 5-U BTX-A-treated group $(P=0.002$ and $P=$ 0.038 for the saline- and 5-U BTX-A-treated groups, respectively).

\section{Discussion}

BTX-A has been used in a variety of oral and maxillofacial applications. BTX-A is primarily injected into masticatory muscles, such as the masseter muscle or the temporal muscle, for esthetic and therapeutic purposes in the maxillofacial area $[3,6]$. A small number of studies that investigated the effects of BTX on masticatory muscles have been reported $[7,17]$. However, short-term molecular-level changes in the masseter muscle after BTX-A injection have not been reported previously. In this study, p65 and S100 were increased in a dosedependent manner in the rat masseter muscle 14 days after the BTX-A injection (Figs. 3 and 4). As the muscle underwent atrophic changes (Fig. 2), the thickness of the ramal cortical bone decreased 14 days after the BTX-A injection (Fig. 1). To our knowledge, this is the first report of dose-dependent early change in the masseter muscle after BTX-A injection.

In this study, the thickness of the cortical bone in the mandibular ramus was decreased 14 days after BTX-A injection in comparison to the saline-treated control (Fig. 1). However, the difference observed among the groups was not statistically significant (Fig. 1d, $P>0.05$ ). Previous publication demonstrates significant difference in bone thickness at 4 and 8 weeks after BTX-A injection [24]. This lack of significance might be due to the small sample size and short follow-up period. The thinner cortex of the mandibular ramus in the BTX-injected group might be similar to the disuse atrophy that occur secondary to masseter muscle atrophy. A number of previous studies reported that if the bone does not receive continuous stimulation from muscles, the bone will atrophy $[8,14]$. When the patient having osteoporosis receives BTX-A injection, the optimal dosage of BTX-A should be carefully monitored. BTX-A injection results in an immediate reduction of electromyographic signals in the masseter muscle [5]. In this study, BTX-Ainjected myofilaments exhibited a reduced size in comparison to the saline-injected control (Fig. 2).

Generally, muscle atrophy is induced by systemic illnesses that contribute to muscle wasting. In this study, BTX-A injection reduced the size of myofibrils; this change should be interpreted as muscle atrophy (Fig. 2). A component of NF-kB (p65) is activated during cytokineinduced myotubule atrophy [25]. In addition, serum S100 induces myoblast apoptosis via the stimulation of reactive oxygen species [26]. In this study, the expression of NF- $k B$ and the S100 was increased in the BTX-A injected groups in comparison to the saline-treated control. When the apoptosis of cells occurs, the expression of NF-kB is increased $[14,27]$. When the calcium concentration in the tissue is increased, the expression of $\mathrm{S} 100$ also is increased $[28,29]$. The observed increase in both p65 and S100 might be caused by muscle atrophy or apoptotic stress after BTX-A injection. When muscle apoptosis occurs, intracellular calcium ions may be released into the extracellular space [30]. As S100 is a calcium-binding protein, increased calcium release may increase the expression of S100. However, S100 protects against myoblast apoptosis [31]. Thus, increased expression of S100 might occur due to the protection of myoblasts from apoptosis after BTXA injection. BTX-A induced myoblast apoptosis is also confirmed in recent publication [32].

\section{Conclusions}

In this study, the injection of BTX-A into the masseter muscle induced muscle atrophy. Subsequently, p65 and S100 expression in myoblasts were increased for the protection of muscle cells.

\section{Acknowledgements \\ This work was carried out with the support of "Cooperative Research Program for Agriculture Science and Technology Development (Project No. PJ01121404)," Rural Development Administration, Republic of Korea. The authors appreciated Jang-Ha Lee and Min-Keun Kim for their help in the animal experiment.}

\section{Authors' contributions}

KSG conducted most of the experiment, and JYY designed the experiment KSG and PYW wrote the manuscript and performed a critical review on the experimental process. All authors read and approved the final manuscript.

Competing interests

The authors declare that they have no competing interests.

\section{Ethics approval and consent to participate}

All procedures were conducted according to the guidelines of laboratory animal care and were approved by the Gangneung-Wonju National University for animal research (GWNUA-2015-25).

\section{Author details}

${ }^{1}$ Department of Oral and Maxillofacial Surgery, College of Dentistry, Gangneung-Wonju National University, 7 Jukhyun-gil, Gangneung 210-702, South Korea. ${ }^{2}$ Sericultural and Apicultural Materials Division, National Academy of Agricultural Science, Suwon, South Korea.

Received: 4 July 2016 Accepted: 22 August 2016

Published online: 26 August 2016

References

1. Burgen A, Dickens F, Zatman L (1949) The action of botulinum toxin on the neuro-muscular junction. J Physiol 109:10-23

2. Schiavo $G$, Benfenati $F$, Poulain B, Rossetto $O$, Polverino de Laureto $P$, DasGupta BR et al (1992) Tetanus and botulinum-B neurotoxins block neurotransmitter release by proteolytic cleavage of synaptobrevin. Nature 359:832-835

3. Su JZ, Cai ZG, Yu GY (2015) Microvascular autologous submandibular gland transplantation in severe cases of keratoconjunctivitis sicca. Maxillofac Plast Reconstr Surg 37:5 
4. Navarrete AL, Rafferty KL, Liu ZJ, Ye W, Greenlee GM, Herring SW (2013) Botulinum neurotoxin type $A$ in the masseter muscle: effects on incisor eruption in rabbits. Am J Orthod Dentofacial Orthop 143:499-506

5. Park SY, Park YW, Ji YJ, Park SW, Kim SG (2015) Effects of a botulinum toxin type $A$ injection on the masseter muscle: an animal model study. Maxillofac Plast Reconstr Surg 37:10

6. Seok H, Lee SW, Kim MK, Kim SG, Park YW, Park SW et al (2013) Correction of post-traumatic lower lip asymmetry using botulinum toxin type A. J Korean Assoc Maxillofac Plast Reconstr Surg 35:256-259

7. Ansved T, Odergren T, Borg K (1997) Muscle fiber atrophy in leg muscles after botulinum toxin type A treatment of cervical dystonia. Neurology 48:1440-1442

8. Kün-Darbois J-D, Libouban H, Chappard D (2015) Botulinum toxin in masticatory muscles of the adult rat induces bone loss at the condyle and alveolar regions of the mandible associated with a bone proliferation at a muscle enthesis. Bone 77:75-82

9. Tsai CY, Lei YY, Yang LY, Chiu WC (2015) Changes of masseter muscle activity following injection of botulinum toxin type $A$ in adult rats. Orthod Craniofac Res 18:202-211

10. Blitzer A, Brin MF, Greene PE, Fahn S (1989) Botulinum toxin injection for the treatment of oromandibular dystonia. Ann Otol Rhinol Laryngol 98:93-97

11. Kim HS, Yun PY, Kim YK (2016) A clinical evaluation of botulinum toxin-A injections in the temporomandibular disorder treatment. Maxillofac Plast Reconstr Surg 38:5

12. von Lindern JJ, Niederhagen B, Appel T, Bergé S, Reich RH (2001) Type A botulinum toxin for the treatment of hypertrophy of the masseter and temporal muscles: an alternative treatment. Plast Reconstr Surg 107:327-332

13. Duchen L, Strich SJ (1968) The effects of botulinum toxin on the pattern of innervation of skeletal muscle in the mouse. Q J Exp Physiol Cogn Med Sci 53:84-89

14. Arora R, Yates C, Gary BD, McClellan S, Tan M, Xi Y et al (2014) Panepoxydone targets NF-kB and FOXM1 to inhibit proliferation, induce apoptosis and reverse epithelial to mesenchymal transition in breast cancer. PLoS One 9(6):e98370

15. Warden SJ, Galley MR, Richard JS, George LA, Guildenbecher EA, Judd AM et al (2013) Reduced gravitational loading does not account for the skeletal effect of botulinum toxin-induced muscle inhibition suggesting a direct effect of muscle on bone. Bone 54:98-105

16. von Lindern JJ, Niederhagen B, Bergé S, Appel T (2003) Type A botulinum toxin in the treatment of chronic facial pain associated with masticatory hyperactivity. J Oral Maxillofac Surg 61:774-778

17. Moon YM, Kim YJ, Kim MK, Kim SG, Kweon HY, Kim TW (2015) Early effect of Botox-A injection into the masseter muscle of rats: functional and histological evaluation. Maxillofac Plast Reconstr Surg 37:46

18. Ilg EC, Schäfer BW, Heizmann CW (1996) Expression pattern of S100 calcium-binding proteins in human tumors. Int J Cancer 68:325-332

19. Mischke D, Korge BP, Marenholz I, Volz A, Ziegler A (1996) Genes encoding structural proteins of epidermal cornification and S100 calcium-binding proteins form a gene complex ("epidermal differentiation complex") on human chromosome 1q21. J Invest Dermatol 106:989-992

20. Heatwole VM (1999) TUNEL assay for apoptotic cells. Immunocytochemical Methods and Protocols. Springer, Heidelberg, p 141-148

21. Wu SH, Huang SH, Cheng Kl, Chai CY, Yeh JL et al (2015) Third-degree hindpaw burn injury induced apoptosis of lumbar spinal cord ventral horn motor neurons and sciatic nerve and muscle atrophy in rats. BioMed Res Int 2015:372819

22. Magnusson-Lind A, Davidsson M, Silajdžić E, Hansen C, McCourt AC, Tabriz SJ et al (2014) Skeletal muscle atrophy in r6/2 mice-altered circulating skeletal muscle markers and gene expression profile changes. J Huntingtons Dis 3:13-24

23. Kim HC, Song JM, Kim CJ, Yoon SY, Kim IR, Park BS et al (2015) Combined effect of bisphosphonate and recombinant human bone morphogenetic protein 2 on bone healing of rat calvarial defects. Maxillofac Plast Reconstr Surg 37:16

24. Vegger JB, Brüel A, Thomsen JS (2015) Vertical trabeculae are thinned more than horizontal trabeculae in skeletal-unloaded rats. Calcif Tissue Int 97:516-526

25. Yamaki T, Wu CL, Gustin M, Lim J, Jackman RW, Kandarian SC (2012) Rel A/p65 is required for cytokine-induced myotube atrophy. Am J PhysiolCell Physiol 303:C135-C142
26. Sorci G, Riuzzi F, Agneletti AL, Marchetti C, Donato R (2004) S100B causes apoptosis in a myoblast cell line in a RAGE-independent manner. J Cell Physiol 199:274-283

27. Seo BN, Ryu JM, Yun SP, Jeon JH, Park SS, Oh KB et al (2013) Delphinidin prevents hypoxia-induced mouse embryonic stem cell apoptosis through reduction of intracellular reactive oxygen species-mediated activation of JNK and NF-kB, and Akt inhibition. Apoptosis 18:811-824

28. Heizmann CW (2013) The calcium signal is transmitted into an intracellular response via the families of calcium-binding proteins which are thought to be involved in the regulation of many cellular activities. Calcium Transport and Intracellular Calcium Homeostasis, vol 48. NATO Advanced Research Workshop, Lyon, p 301

29. Mishra SK, Siddique HR, Saleem M (2012) S100A4 calcium-binding protein is key player in tumor progression and metastasis: preclinical and clinical evidence. Cancer Metastasis Rev 31:163-172

30. Yu SP, Canzoniero LM, Choi DW (2001) Ion homeostasis and apoptosis. Curr Opin Cell Biol 13:405-411

31. Riuzzi F, Sorci G, Donato R (2006) S100B stimulates myoblast proliferation and inhibits myoblast differentiation by independently stimulating ERK1/2 and inhibiting p38 MAPK. J Cell Physiol 207:461-470

32. Moon YM, Kim MK, Kim SG, Kim TW (2016) Apoptotic action of botulinum toxin on masseter muscle in rats: early and late changes in the expression of molecular markers. Springerplus 5:991

\section{Submit your manuscript to a SpringerOpen ${ }^{\circ}$ journal and benefit from:}

- Convenient online submission

- Rigorous peer review

- Immediate publication on acceptance

- Open access: articles freely available online

- High visibility within the field

- Retaining the copyright to your article

Submit your next manuscript at $>$ springeropen.com 